Pacific Journal of Mathematics

LOCALLY COMPACT CLIFFORD SEMIGROUPS 


\title{
LOCALLY COMPACT CLIFFORD SEMIGROUPS
}

\author{
J. W. STEPP
}

Let $S$ be a locally compact Hausdorff semigroup which is a disjoint union of subgroups one of which is dense. If $S$ the disjoint union of exactly two groups one of which is compact, then $S$ has been completely described by K. H. Hofmann, and if $S$ is the disjoint union of two subgroups where the dense subgroup $G$ has the added property that it is abelian and $G / G_{0}$ is a union of compact groups, then $S$ has been described in a previous paper of the author.

It is the purpose of this paper to consider $S$ when each subgroup of $S$ is a topological group when given the relative topology and $G$ (the dense subgroup) has the added property that it is abelian and $G / G_{0}$ is a union of compact groups. In particular, we show how to reduce such a semigroup to a semigroup which is a union of real vector groups $(\S 3)$. In $\S 4$ we give the structure of $S$ under the added assumption that $E(S)$ is isomorphic to $E\left[\left(R^{x}\right)^{n}\right]$, where $\left(R^{x}\right)^{n}$ denotes the $n$-fold product of the nonnegative real numbers under multiplication.

2. Definitions and notations. If $G$ is a topological group, $G_{0}$ will denote the identity component. Let $\mathscr{C}$ denote the full subcategory of the category of locally compact abelian groups whose objects $G$ have the property that $G / G_{0}$ is a union of compact subgroups. Let $\mathscr{C}_{c}$ denote the full subcategory of $\mathscr{C}$ whose objects $G_{c}$ have the property that $G_{c}$ is a union of compact subgroups. If $G \in \mathscr{C}$, then by the structure theorem for locally compact abelian groups [2, p. 389] there is a real vector subgroup $W$ of $G$ such that $G / W \in \mathscr{C}_{c}$. If $W \cong R^{n}$, then $n=\operatorname{dim} G$ will be called the dimension of $G$. We will use the following properties of $\mathscr{C}$ and $\mathscr{C}_{c}: P_{1}$; for each $G$ in $\mathscr{C}$ there is a unique subgroup $G_{c} \in \mathscr{C}_{c}$ such that $G / G_{c}$ is a real vector group. $P_{2}$ [7]; if $\alpha: G \rightarrow W$ is a morphism in $\mathscr{C}$ with $\alpha(G)$ dense in $W$ and if $W$ is a real vector group, then there is morphism $\beta: W \rightarrow G$ in $\mathscr{C}$ such that $\alpha \beta=I_{W}$ (the identity morphism on $W$ ). $P_{3}$ [7]; if $\alpha: G \rightarrow H$ is a morphism in the category of locally compact abelian groups with $\alpha(G)$ dense in $H$ and $G \in \mathscr{C}$, then $H \in \mathscr{C}$. Also, if $G / G_{0}$ is compact, then $H / H_{0}$ is compact.

Let $\mathscr{S}$ denote the category whose objects $S$ are locally compact Hausdorff semigroups satisfying (i) $S$ is a disjoint union of subgroups one of which is dense and (ii) each maximal subgroup of $S$ is a member of $\mathscr{C}$, and whose morphisms are the continuous identity preserving homomorphisms. Let $\mathscr{R}$ denote the full subcategory of $\mathscr{S}$ whose objects $R$ have the properties that (i) each maximal subgroup of $R$ 
is a real vector group and (ii) the minimial ideal of $R$ exists and is compact (thus a zero for $R$ ).

Let $S \in \mathscr{S}$. Then we will use 1 to denote the identity for $S$. For each $x$ in $S$ let $H(x)=\{y \in S \mid y S=x S\}$. Since $S$ is an abelian Clifford semigroup, each $H(x)$ is a maximal subgroup of $S$. Let $\delta: S \rightarrow E(S)$ be the function defined by $\delta(s)$ is the idempotent of $S$ such that $H(s)=H(\delta(s))$. If $A \subseteq S$, then $\bar{A}$ will denote the closure of $A$. Partially order $E(S)$ by $e \leqq f$ if and only if $e f=e$, and for each $e$ and $f$ in $E(S)$ let $(e, f)=\{a \in E(S) \mid e<a<f\}$. Let $Z=\{0,1\}$ under multiplication, and let $Z^{n}$ denote the $n$-fold product of $n$ copies of $Z$. Finally, for a semigroup $T$ we use $K(T)$ to denote the minimial ideal when it exists.

3. The purpose of this section is two fold. First we prove that each $S$ in $\mathscr{S}$ splits into the direct product of two closed subsemigroups $V$ and $\bar{W}$, where $V$ is a real vector group and where $\bar{W} \in \mathscr{S}$ with the added property that $K(\bar{W}) \in \mathscr{C}_{c}$ (Proposition 3.5). Second we prove that there is a congruence $\rho$ on $S$ such that $S / \rho$ is a locally compact Clifford semigroup with each $H$-class a real vector group and with $E(S) \cong E(S / \rho)$ (Theorem 3.11).

Throughout this section $S$ will represent a fixed member of $\mathscr{S}$, and $E(S)^{*}$ will denote $E(S) \backslash\{1\}$.

Lemma 3.1. Let $e \in E(S)^{*}$. Then $H(e)$ is open in $S \backslash H(1)$ if and only if $\operatorname{dim} H(e)=\operatorname{dim} H(1)-1$.

Proof. By [7], if $H(e)$ is open in $S \backslash H(1)$, then $\operatorname{dim} H(e)=$ $\operatorname{dim} H(1)-1$.

Let $e \in E(S)$ with $\operatorname{dim} H(e)=\operatorname{dim} H(1)-1$. Again by [7], if $f \in E(S)$ such that $e<f$, then $\operatorname{dim} H(e)<\operatorname{dim} H(f)$. Thus, since $\operatorname{dim} H(f)<\operatorname{dim} H(1)$ for all $f$ in $E(S)^{*}$ [7], $(e, 1)=\varnothing$. Let $\psi: S \rightarrow e S$ be the morphism defined by $\psi(s)=e s$. Since $H(e)$ is a topological group, $H(e)$ is open in $\overline{H(e)}[8]$ which is $e S$. Since $\psi$ is continuous. and since $H(e)=(S \backslash H(1)) \cap \psi^{-1}(H(e))$, it follows that $H(e)$ is open in. $S \backslash H(1)$.

CoROLlary 3.2. If $e \in E(S)^{*}$, then there is an $f$ in $E(S)$ with $e<f$ and $\operatorname{dim} H(e)=\operatorname{dim} H(f)-1$.

Proof. Let $f \in E(S)$ with $e<f$ and $(e, f)=\varnothing$. Then $H(e) \subseteq \overline{H(f)}$. Let $\psi: \overline{H(f)} \rightarrow e \overline{H(f)}$ morphism defined by $\psi(s)=e s$. Since $(e, f)=\varnothing$, $H(e)=\overline{(H(f)} \backslash H(f)) \cap\left(\psi^{-1}(H(e))\right)$, and it follows that $H(e)$ is open in. $\overline{H(f)} \backslash H(f)$. Thus, by Lemma 3.1, $\operatorname{dim} H(e)=\operatorname{dim} H(f)-1$. 
Lemma 3.3. A subgroup $H \in \mathscr{C}_{\text {c }}$ of $S$ is closed in $S$.

Proof. Let $g \in \bar{H}$. Since $H \leqq H(e)_{c}$ for some $e$ in $E(S)$ and $g \in \overline{\delta(g) H}$, it follows that $g \in H(g)_{c}$. Thus there is a compact subgroup $C$ of $H(g)_{c}$ with $g \in C$. Since $\left\{g^{n}\right\}_{n=1}^{\infty} \subseteq C$ and $C$ is compact, $\delta(g) \in \overline{\left\{g^{n}\right\}_{n=1}^{\infty}}$ [4, p. 15] which is a subset of $\bar{H}$; thus $\delta(g) \in \bar{H}$. By [7], there are no maximal subgroups of $\bar{H}$ which are topological other than $\bar{H}$; thus $\delta(g)=e$, and $\bar{H} \subseteq H(e)$. Thus we need only show that $H$ is a closed subgroup of $H(e)$, but this follows since $H$ is a locally compact subgroup of a locally compact topological group.

Proposition 3.4. Let $e \in E(S)$, and let $\psi$ be the map from $S$ onto $e S$ defined by $\psi(s)=e s$. Then there are closed subgroups $V$ and $W$ of $H(1)$ with the following properties:

(a) $\bar{W}=\psi^{-1}\left(H(e)_{c}\right)$,

(b) $V$ is a real vector group, and

(c) The morphism $m: V \times \bar{W} \rightarrow \psi^{-1}(H(e))$ defined by $m(v, w)=v \cdot w$ is an isomorphism.

Proof. Let $\alpha$ be the natural map from $H(e)$ onto $H(e) / H(e)_{c}$, let $Q$ be the corestriction of $\left.\psi\right|_{H(1)}$ to $H(e)$, and let $\beta: H(e) / H(e)_{c} \rightarrow H(1)$ be a morphism in $\mathscr{C}$ such that $(\alpha Q) \beta$ is the identity map on $H(e) / H(e)_{c}$ $\left[P_{2}\right]$. Let $V=\beta\left(H(e) / H(e)_{c}\right)$, and let $W=Q^{-1}\left(H(e)_{c}\right)$. Then $V$ and $W$ are the desired closed subgroups of $H(1)$. The inverse of $m$ is given by $s \mapsto\left((\beta \alpha \psi)(s),[(\beta \alpha \psi)(s)]^{-1} s\right)$ which is clearly continuous. The theorem now follows.

Proposition 3.5. There are closed subgroups $V$ and $W$ of $H(1)$ with the following properties:

(a) $V$ is a real vector group,

(b) $K(\bar{W}) \in \mathscr{C}_{c}$, and

(c) The morphism $m: V \times \bar{W} \rightarrow S$ defined by $m(v, w)=v \cdot w$ is an isomorphism.

Proof. Again by [7], if $e \in E(S)^{*}$, then $\operatorname{dim} H(e)<\operatorname{dim} H(1)$. Thus there is an $f$ in $E(S)$ with $\operatorname{dim} H(f) \leqq \operatorname{dim} H(e)$ for all $e$ in $E(S)$. Since $\operatorname{dim} H(e f) \leqq \min \{\operatorname{dim} H(e), \operatorname{dim} H(f)\}$ with equality holding only for $e<f$ or $f \leqq e, f$ is unique. The proposition now follows from Proposition 3.4 along with the observation that $S=\psi^{-1}(H(f))$ where $\psi: S \rightarrow f S$ is the morphism defined by $\psi(s)=s f$ for all $s$ in $S$.

Proposition 3.6. If there is a $s_{0}$ in $S$ with $H\left(s_{0}\right)_{c}$ compact, then $H(s)_{c}$ is compact for all $s$ in $S$. 
Proof. From the structure theorem for locally compact abelian groups [2, p. 389] one can get that if $G \in \mathscr{C}_{c}$, then $G_{0}$ is compact. Thus for any $s$ in $S$ we have that $H(s)_{c}$ is compact if and only if $H(s)_{c} /\left(H(s)_{c}\right)_{0}$ is compact. But $\left.H(s)_{c} / H(s)_{c}\right)_{0}$ is compact if and only if $H(s) / H(s)_{0}$ is compact. Therefore, by $P_{3}$ and since $\overline{H(1)}=S$, the theorem will follow if we can prove that $H(1) / H(1)_{0}$ is compact.

We do this by contradiction. That is, assume $H(1) / H(1)_{0}$ is not compact, and let $e \in E(S)$ satisfying the following:

(i) $H(e) / H(e)_{0}$ is compact,

(ii) $\delta\left(s_{0}\right) \leqq e$, and

(iii) if $f \in E(S)$ with $e<f$, then $H(f) /(f)_{0}$ is not compact.

By Corollary 3.2 and since $e \neq 1$, there is an $f$ in $E(S)$ with $e<f$ and $\operatorname{dim} H(e)=\operatorname{dim} H(f)-1$. Let $T=\overline{H(f)}$, and let $\psi: T \rightarrow e T$ be the morphism defined by $\psi(s)=s e$. By Proposition 3.4, there is a real vector subgroup $V$ of $H(f)$, a closed subgroup $W$ of $H(f)$ with $\psi^{-1}\left(H(e)_{c}\right)=\bar{W}$, and a morphism $m: V \times \bar{W} \rightarrow \psi^{-1}(H(e))$ which is an isomorphism. Since $\bar{W} \backslash W=H(e)_{c}$ which is compact and by [3], $W$ contains a compact subgroup $C$ such that $W / C$ is a real vector group. Thus $H_{W}(f)_{c}$ is compact. Since the corestriction of $\left.m\right|_{V \times W}: V \times W \rightarrow H(f)$ is an isomorphism and $V$ is a real vector group, it now follows that $H(f)_{c}$ is compact. This is the desired contradiction and the proof now follows.

Sublemma. Let $e$ and $f$ be elements of $E(S)$ with $\operatorname{dim} H(e)=$ $\operatorname{dim} H(f)+1$ and with $f<e$. If $H$ is a subgroup of $H(e)$ with $H \in \mathscr{C}_{c}$, then $f H$ is a closed subgroup of $S$.

Proof. Let $g \in \overline{f H} \cap H(f)$. Since $H \in \mathscr{C}_{c}, f H \subseteq H(f)_{c}$, and thus there is a compact subgroup $C$ of $H(f)$ which is open relative to $H(f)_{c}$ and with $g \in C$. Let $\psi: \overline{H(e)} \rightarrow \overline{f H(e)}$ be the morphism defined by $\psi(s)=f s$. It follows from Proposition 3.4 and the fact that $H(f)$ is open in $\overline{H(e)} \backslash H(e)$ that $\psi^{-1}(C)$ is a locally compact semigroup which contains a dense group $\psi^{-1}(C) \cap H(e)$ whose complement $C$ is compact. By [3], there is a unique compact subgroup $C_{1}$ of $\psi^{-1}(C) \cap H(e)$ and a one-parameter subgroup $M$ of $\psi^{-1}(C) \cap H(e)$ such that $\psi^{-1}(C)=\bar{M} \cdot C_{1}$. Let $\left\{g_{\alpha}\right\}_{\alpha \in A}$ be a net in $f H$ which converges to $g$. Since $C$ is open in $H(f)_{c}$, there is a $\beta \in A$ such that if $\alpha \geqq \beta$, then $g_{\alpha} \in C$. For each $\alpha \in A$ with $\alpha \geqq \beta$ there is an $h_{\alpha} \in H$ with $g_{\alpha}=f h_{\alpha}$. It follows that each $h_{\alpha} \in C_{1}$, and therefore there is an $h$ in $C_{1} \cap H$ such that $f h=g$. Thus $\overline{f H} \subseteq f H \subseteq \overline{f H}$. We now have $f H$ is a closed subgroup of $H(f)_{c}$, and therefore $f H \in \mathscr{C}_{c}$. The sublemma now follows by Lemma 3.3.

Lemma 3.7. If $H$ is a subgroup of $S$ with $H \in \mathscr{C}_{c}$ and if $f \in E(S)$, then $f H$ is closed. 
Proof. Let $h \in H$; then $\delta(h) \cdot f \leqq \delta(h)$. If $\delta(h) f=\delta(h)$, then $f H=$ $f \delta(h) H=\delta(h) H=H$ which is closed by Lemma 3.3. If $\delta(h) \cdot f<\delta(h)$, then there is a chain of idempotents $e_{1} \cdots, e_{q+1}$ which is maximal with respect to the properties: (i) $e_{1}=\delta(h) f$ and (ii) $e_{q+1}=\delta(h)$. Observe that since $e_{1}, \cdots, e_{q+1}$ is maximal, $\operatorname{dim} H\left(e_{i}\right)=\operatorname{dim} H\left(e_{i+1}\right)-1$ for $i=$ $1,2, \cdots, q$. If $f H$ is not closed, then there is an integer $p, 1 \leqq p \leqq q$ such that $e_{p} H$ is not closed and $e_{p+1} H$ is closed. Since $e_{p} H=\left(e_{p} \cdot e_{p+1}\right) H=$ $e_{p}\left(e_{p+1} H\right)$ and since $e_{p+1} H \in \mathscr{C}_{c}, e_{p} H$ is closed (sublemma). Thus $e_{p} H$ is both closed and not closed which is impossible; thus it follows that $f H$ must be closed.

Now that one has Lemma 3.7 it is easy to prove the following corollary.

CoRollary 3.8. (i) For each $x$ in $S, x H(1)_{c}$ is closed.

(ii) If $U$ is a nonempty compact subset of $S$, then $U \cdot H(1)_{c}$ is closed.

THeOREM 3.9. Let $R=\left\{(x, y) \in S \times S \mid x H(1)_{c}=y H(1)_{c}\right\}$. Then $R$ is a congruence, and $S / R$ is a locally compact semigroup with the following properties:

(i) If $\theta$ is the natural map from $S$ onto $S / R$, then $\theta$ is an open map and $\theta(H(s)) \cong H(s) /\left(\delta(s) H(1)_{c}\right)$ for all $s$ in $S$.

(ii) The corestriction of $\left.\theta\right|_{E(S)}$ to $E(S / R)$ is an isomorphism.

Proof. Clearly $R$ is a congruence. Since $H(1)$ acts as a group of homeomorphisms on $S$ and since $\theta^{-1}(\theta(A))=A \cdot H(1)$ c for all $A \neq \varnothing$, it follows that $\theta$ is an open map. Since $\theta$ is an open map, $S / R$ is locally compact and also multiplication is continuous. We now show $S / R$ is Hausdoff. Let $x, y \in S$ with $x H(1)_{c} \neq y H(1)_{c}$. Since $y H(1)_{c}$ is closed (Corollary 3.8) and since $S$ is a locally compact (thus regular) Hausdorff space, there is a compact neighborhood $N_{x}$ of $x$ with $N_{x} \cap y H(1)_{c}=\varnothing$. Thus $y \notin N_{x} \cdot H(1)_{c}$ which is closed by Corollary 3.8, and using the fact that $S$ is regular we obtain a compact neighborhood $N_{y}$ of $y$ with $N_{y} \cap\left(N_{x} \cdot H(1)\right)_{c}=\varnothing$. It follows that $\left(N_{y} \cdot H(1)_{c}\right) \cap$ $\left(N_{x} \cdot H(1)_{c}\right)=\varnothing$, and thus $S / R$ is Hausdorff. This completes the proof.

REMARK. We wish to point out that each maximal subgroup of $S / R$ is connected, and thus $H(\theta(s))_{c}$ is compact for each $s$ in $S$.

Lemma 3.10. Let $T \in \mathscr{S}$ with $K(T)$ compact. Then for each nonnegative integer $n$ there is a $T_{n}$ in $\mathscr{S}$ and a surmorphism $\alpha_{n}: T \rightarrow T_{n}$ in $\mathscr{S}$ satisfying:

(a) The corestriction of $\left.\alpha_{n}\right|_{E(S)}$ to $E\left(T_{n}\right)$ is an isomorphism.

(b) If $x \in T$ with $\operatorname{dim} H(x) \leqq n$, then $\alpha_{n}(H(x))=H\left(\alpha_{n}(x)\right) \cong H(x) / H(x)_{c}$. 
(c) If $x \in T$ with $\operatorname{dim} H(x)>n$, then the corestriction of $\left.\alpha\right|_{H(x)}$ to $H(\alpha(x))$ is an isomorphism.

Proof. The proof is by induction. Let $R_{0}=\{(x, y) \mid x=y$ or $x \in K(T)$ and $y \in K(T)\}$. Clearly $R_{0}$ is a congruence, and since $K(T)$ is compact, it follows that $T / R_{0}$ is a locally compact semigroup. Let $\alpha_{0}$ be the natural map from $T$ onto $T / R_{0}=T_{0}$. Then, clearly, $\alpha_{0}$ and $T_{0}$ satisfy (a)-(c) for $n=0$.

Let $k$ be a nonnegative integer such that there is a $T_{k} \in \mathscr{S}$ and a surmorphism $\alpha_{k}: T \rightarrow T_{k}$ satisfying (a)-(c). If $k \geqq \operatorname{dim} H(1)$, then let $T_{k+1}=T_{k}$ and $\alpha_{k+1}=\alpha_{k}$. Then $T_{k+1}$ and $\alpha_{k+1}$ satisfy (a)-(c). If $k<\operatorname{dim} H(1)$, let $A=\left\{e \in E\left(T_{k}\right) \mid \operatorname{dim} H(e)=k+1\right\}$, and let $\hat{T}_{k}=$ $\left\{x \in T_{k} \mid x \in \overline{H(e)}\right.$ for some $e$ in $\left.A\right\}$. For each $e$ in $A$ let $\psi_{e}: S \rightarrow e S$ be the morphism defined by $\psi_{e}(s)=e s$. Then $\psi_{e}^{-1}(H(e)) \cap \hat{T}_{k}=H(e)$, and thus each $H(e)$ is open relative to $\hat{T}_{k}$. Let $R_{k+1}=\left\{(x, y) \in T_{k} \times T_{k} \mid x=y\right.$ or $\delta(x)=\delta(y) \in A$ and $\left.x \in y H(\delta(y))_{c}\right\}$. It is easy to show that $R_{k+1}$ is a congruence. By Proposition 3.6 and since $K\left(T_{k}\right)=\{0\}$, each $H(e)_{c}$ is compact. Since each $H(e)_{c}$ is compact and since each $H(e)$ with $e \in A$ is open in $\widehat{T}_{k+1}$, it follows that $T_{k} / R_{k+1}$ is a locally compact semigroup. Let $T_{k+1}=T_{k} / R_{k+1}$ and $\alpha_{k+1}=\eta \alpha_{k}$, where $\eta$ is the natural map from $T_{k}$ onto $T_{k} / R_{k+1}$. Then $T_{k+1} \in \mathscr{S}$ and $\alpha_{k+1}: T \rightarrow T_{k+1}$ is a surmorphism satisfying (a)-(c) for $n=k+1$. The theorem now follows by induction.

THEOREM 3.11. Let $S \in \mathscr{S}$. Then there is a $T \in \mathscr{S}$ and a surmorphism $\alpha: S \rightarrow T$ in $\mathscr{S}$ satisfying:

(i) The corestriction of $\left.\alpha\right|_{E(S)}$ onto $E(T)$ is an isomorphism.

(ii) Each $H$-class of $T$ is a real vector group.

Proof. By Proposition 3.5, there is an isomorphism $\beta: S \rightarrow V \times \bar{T}$ where $V$ is a real vector group and where $\bar{T} \in \mathscr{S}$ with $K(\bar{T}) \in \mathscr{C}_{\text {c }}$. By first applying Theorem 3.9 and then Lemma 3.10 for $n=\operatorname{dim} H(1)$ one can obtain a surjective morphism $\beta_{1}: \bar{T} \rightarrow T_{n}$ which preserves the structure of $E(\bar{T})$ and where the $H$-class of $T_{n}$ are real vector groups. Let $T=V \times T_{n}$ and $\alpha: S \rightarrow V \times T_{n}$ be the map defined by $\alpha(s)=$ $\left(p_{r_{1}}(\beta(s)), \beta_{1}\left(p_{r_{2}}(\beta(s))\right)\right.$. Then clearly $T$ and $\alpha: S \rightarrow T$ satisfy the conditions of the theorem.

4. Let $\mathscr{S}_{1}$ denote the full subcategory of $\mathscr{S}$ whose objects $S$ have the property that $E(S) \cong Z^{q}$ for some nonnegative integer $q$. In this section we characterize the objects in $\mathscr{S}_{1}$. The fact that there are objects in $\mathscr{S}$ that are not in $\mathscr{S}_{1}$ is demonstrated by J. G. Horne, $\mathrm{Jr}$., in [6]. However, if $S \in \mathscr{S}$ with $\operatorname{dim} H(1) \leqq 2$, then it is shown 
that $S \in \mathscr{S}_{1}$.

Let $R_{+}$denote the multiplicative group of positive real numbers, and recally that $R^{x}$ denotes the multiplicative semigroup of nonnegative real numbers.

LEMma 4.1. Let $E$ be a Hausdoff topological space which is the disjoint union of $R_{+} \times R^{x}$ and a singleton set $\{w\}$, where $R_{+} \times R^{x}$ has the product topology. If $\{w\} \cup\left(R_{+} \times\{0\}\right)$ is homeomorphic to $R^{x}$ with $\overline{w \in(0,1] \times\{0\}}$, then $E$ is not locally compact at $w$.

Proof. We assume $E$ is locally compact at $w$ and show that this assumption leads to the conclusion that $R^{x}$ is compact. Let $U$ be an open neighborhood of $w$ with $\bar{U}$ compact. Then $\bar{U} \backslash U$ is a compact subset of $R_{+} \times R^{x}$. Since $w \cup\left(R_{+} \times\{0\}\right)$ is homeomorphic to $R^{x}$ with $\overline{(0,1] \times\{0\}}=((0,1] \times\{0\}) \cup\{w\}$, there is an $a$ in $R_{+}$with $\{(x, 0) \mid 0<x<a\} \subseteq U$. For each $b$ in $R_{+}$with $0<b<a$ either $\{b\} \times R^{x} \subseteq U$ or $\left(\{b\} \times R^{x}\right) \cap(\bar{U} \backslash U) \neq \varnothing$. To see this, assume $\left(\{b\} \times R^{x}\right) \cap(\bar{U} \backslash U)=\varnothing$. Then $\{b\} \times R^{x}$ is the disjoint union of the two relatively open sets $(E / \bar{U}) \cap\left(\{b\} \times R^{x}\right)$ and $U \cap\left(\{b\} \times R^{x}\right)$. Since $\{b\} \times R^{x}$ is connected and $\{b\} \times R^{x} \cap U \neq \varnothing,(E \backslash \bar{U}) \cap\left(\{b\} \times R^{x}\right)=\varnothing$ and hence $\{b\} \times R^{x} \subseteq U$.

We now prove there is a $r_{0}<a$ in $R_{+}$satisfying; if $b \in R_{+}$and $b \leqq r_{0}$, then $\{b\} \times R^{x} \subseteq U$. If this were not the case, then by the above there would exists a sequence $\left\{b_{n}\right\}_{n=1}^{\infty}$ in $R_{+}$such that $\left.\left\{b_{n}, 0\right)\right\}_{n=1}^{\infty}$ converges to $w$, and each $\left(\left\{b_{n}\right\} \times R^{x}\right) \cap(\bar{U} \backslash U) \neq \varnothing$. For each positive integer $n$ let $x_{n}$ be an element of $R^{x}$ such that $\left(b_{n}, x_{n}\right) \in \bar{U} \backslash U$. Since $\bar{U} \backslash U$ is a compact subset of $R_{+} \times R^{x}$, the sequence $\left\{\left(b_{n}, x_{n}\right)\right\}_{n=1}^{\infty}$ has a cluster point $(b, x)$. Thus $\left\{\left(b_{n}, 0\right)\right\}_{n=1}^{\infty}$ converges to $w$ and clusters to $(b, 0)$ which is impossible. Thus we now can conclude that there is a $r_{0}$ in $R_{+}$such that if $b \in R_{+}$with $b \leqq r_{0}$, then $\{b\} \times R^{x} \subseteq U$. We point out at this point that if $b \in R_{+}$and $b \leqq r_{0}$, then $\{b\} \times R^{x}=\{w\} \cup\{b\} \times R^{x}$.

For each $l$ in $R_{x},\left\{(r, l) \mid r_{0} \leqq r\right\}$ is connected, and $\left(r_{0}, l\right) \in U$. Thus a similar argument to the one above proves there is an $l_{0}$ in $R^{x}$ such that if $l \geqq l_{0}$ then $\left\{(r, l) \mid r_{0} \leqq r\right\} \subseteq U$. Similarly, there is a $t_{0} \in R_{+}$with $t_{0} \geqq r_{0}$ and such that if $t \in R_{+}$with $t \geqq t_{0}$, then $\left\{(t, l) \mid 0 \leqq l \leqq l_{0}\right\} \subseteq U$. Let $B=\left[r_{0}, t_{0}\right] \times\left[0, l_{0}\right]$ which is a compact subset of $R_{+} \times R^{x}$. It is easy to show that $E \backslash B \subseteq U$ and thus $E=\bar{U} \cup B$ and is compact. In particular, $(R \times\{0\}) \cup\{w\}$ is compact and homeomorphic to $R^{x}$. This is the desired contradiction.

THEOREM 4.2. If $S$ is a member of $\mathscr{R}$ with $\operatorname{dim} H(1)=2$, then $E(S) \cong Z^{2}$.

Proof. Since $S \in \mathscr{R}, S$ has a zero. By Corollary 3.2, there is an element $f$ in $E(S)$ with $\operatorname{dim} H(f)=1$. 
Case 1. There is only one $f$ in $E(S)$ with $\operatorname{dim} H(f)=1$. That is, $E(S)=\{0, e, 1\} . \quad$ By [7], $S \backslash\{0\} \cong R_{+} \times R^{x}$. By [5] and since $\overline{R_{+} \times\{0\}}=$ $\left(R_{+} \times\{0\}\right) \cup\{0\}, \overline{R \times\{0\}}$ is homeomorphic to $R^{x}$. By applying Lemma 4.1 we have $S$ is not locally compact at $\{0\}$. Thus Case 1 is impossible.

Case 2. There are exactly two idempotents $e_{1}$ and $e_{2}$ with $\operatorname{dim} H\left(e_{1}\right)=\operatorname{dim} H\left(e_{2}\right)=1$. Clearly in this case $E(S) \cong Z^{2}$.

Case 3. There are at least three idempotents $e_{1}, e_{2}, e_{3}$ with $\operatorname{dim} H\left(e_{i}\right)=1$. Let $P_{1}$, and $P_{2}$ be one-parameter subgroups of $H(1)$ with $\bar{P}_{i}=P_{i} \cup\left\{e_{i}\right\}$ (Proposition 3.5). Let $\left\{s_{\alpha}\right\}$ be a net in $H(1)$ which converges to $e_{3}$. Since $S \backslash H(1)$ is an ideal, $\left\{s_{\alpha}^{-1}\right\}$ does not have a cluster point. Since $H(1)=P_{1} \cdot P_{2}$, there are nets $\left\{s_{1 \alpha}\right\} \subseteq P_{1}$ and $\left\{s_{2 \alpha}\right\} \subseteq P_{2}$ such that $s_{1 \alpha} \cdot s_{2 \alpha}=s_{\alpha}$ for all $\alpha$. By [3] and since $\left\{s_{\alpha}^{-1}\right\}$ does not have a cluster point, either $\left\{s_{1 \alpha}\right\}$ clusters to $e_{1}$ and $\left\{s_{2 \alpha}^{-1}\right\}$ clusters to $e_{2}$ or $\left\{s_{1 \alpha}^{-1}\right\}$ clusters to $e_{1}$ and $\left\{s_{2 \alpha}\right\}$ clusters to $e_{2}$. But the former implies $e_{1}=e_{3} \cdot e_{2}$, and the latter implies $e_{2}=e_{1} \cdot e_{3}$. Since $e_{3} \cdot e_{2}=0$ and $e_{1} \cdot e_{3}=0$, either $e_{1}=0$ or $e_{2}=0$. This is the desired contradiction. Thus Case 3 is impossible.

LemMA 4.3. Let $S$ be a member of $\mathscr{R}$ with $\operatorname{dim} H_{S}(1) \geqq 2$, and let $e \in E(S)$ with $H(e) \cong R_{+}$. Then there is an $f$ in $E(S)$, such that $\operatorname{dim} H(f)=\operatorname{dim} H_{S}(1)-1$ and that $e f=0$.

Proof. By Corollary 3.2 and since $\operatorname{dim} H_{S}(1) \geqq 2$, there is an idempotent $e_{1}$ in $S$ such that $e<e_{1}$ and that $\operatorname{dim} H\left(e_{1}\right)=\operatorname{dim} H(e)+1=2$. Let $T=\overline{H\left(e_{1}\right)}$, then $T$ is a member of $\mathscr{R}$ and $\operatorname{dim} H_{T}(1)=2$. Thus, by applying Theorem 4.2 to $T$ one observes that there is an $f$ in $E(T)^{*}$ (and thus in $E(S)^{*}$ ) such that $f \neq 0$ and that $e f=0$. Let $f$ be a maximal such idempotent with respect to $f \neq 0$ and $e f=0$.

Claim. $\operatorname{dim} H(f)=\operatorname{dim} H_{S}(1)-1$. If this were not the case, then applying Corollary 3.2 two time we observe there are idempotents $f_{1}$ and $f_{2}$ such that $f<f_{1}<f_{2}$ and $\operatorname{dim} H(f)=\operatorname{dim} H\left(f_{1}\right)-1=\operatorname{dim} H\left(f_{2}\right)-2$. By applying Proposition 3.4 to $\overline{H\left(f_{2}\right)}$ we observe there is a subsemigroup $R \subseteq \overline{H\left(f_{2}\right)}$ such that $K(R)=\{f\}$ and $\operatorname{dim} H_{R}(1)=2$. By Theorem 4.2, there is an idempotent $f_{3}$ in $E(R)^{*}$ (and thus $E(S)^{*}$ ) such that $f_{3} \neq f$ and $f_{3} \cdot f_{1}=f$. But since $f_{1}$ and $f_{3}$ are elements of $E(S)^{*}$ which are larger that $f, e f_{1} \neq 0$ and $e f_{3} \neq 0$. In fact, since $e f_{1} \leqq e$ and $e f_{3} \leqq e$, $e f_{1}=e f_{2}=e$. However, $0=e f=e\left(f_{1} f_{3}\right)=e f_{3}=e$, and this is the desired contradiction. Therefore, $f$ is maximal in $E(S)^{*}$. From the proof of Lemma 3.1, we have that $f$ maximal in $E(S)^{*}$ implies $\operatorname{dim} H(f)=$ $\operatorname{dim} H(1)-1$. For the remainder of this paper we will use the following notation. If $S \in \mathscr{S}$ and $e \in E(S)$, then $\psi_{e}: S \rightarrow e S$ is the morphism defined by $\psi_{e}(s)=e s$ for all $s$ in $S$. 
We omit the proof of the next lemma since the proof is straight forward.

LEMMA 4.4. (i) If $f$ and $e$ are element of $E\left(\left(R^{x}\right)^{n}\right)$ with $\operatorname{dim} H(f)=1, \operatorname{dim} H(e)=n-1$ and if $\psi_{f}^{-1}(f) \cap \psi_{e}^{-1}(e)=\{1\}$, then the morphism $m: \psi_{f}^{-1}(f) \times \psi_{e}^{-1}(e) \rightarrow\left(R^{x}\right)^{n}$, defined by $m(s, t)=s t$, is an isomorphism.

(ii) If $e \in E(S)$ with $\operatorname{dim} H(e)=p$, then (a) $\psi_{e}^{-1}(e) \cong\left(R^{x}\right)^{n-p}$ and (b) $\psi_{e}\left[\left(R^{x}\right)^{n}\right] \cong\left(R^{x}\right)^{p}$.

LEMMA 4.5. If $\alpha:\left(R^{x}\right)^{n} \rightarrow\left(R^{x}\right)^{n} \in \mathscr{R}$ is a surmorphism with $\alpha\left(E\left(R^{x}\right)^{n}\right)=E\left(\left(R^{x}\right)^{n}\right)$, then $\alpha$ is an isomorphism.

Proof. The proof is by induction on $\operatorname{dim} H(1)$. The lemma is trivially true for $n=0$. If $n=1$, then $\alpha\left(R_{+}\right)$is a dense connected subgroup of $R^{x}$ and thus $\alpha\left(R_{+}\right)=R_{+}$. By [2, p. 84], $\left.\alpha\right|_{R_{+}}: R_{+} \rightarrow R_{+}$ is an isomorphism, and thus it follows that $\alpha$ is bijective. We show $\alpha$ is a closed map. Let $A$ be a closed subset of $R^{x}$. If $A \cong R_{+}$, then there is an $r$ in $R_{+}$with $[0, r] \cap A=\varnothing$. Thus $\alpha(A)$ is closed in $R_{+}$ and $[0, f(r)) \cap \alpha(A) \subseteq[0, f(r)] \cap \alpha(A)=\varnothing$. Since $[0, f(r))$ is open in $R^{x}, 0 \notin \overline{\alpha(A)}$, and thus it follows that $\overline{\alpha(A)}=\alpha(A)$. If $0 \in A$, then either $A=R^{x}$ or there is an $r$ in $R_{+}$with $r \notin A$. If $A=R^{x}$, then clearly $\alpha(A)$ is closed. If there is an $r$ in $R_{+}$with $r \notin A$, then $A=$ $([0, r] \cap A) \cup([r, \infty) \cap A)$. We now have

$$
\begin{aligned}
\alpha(A) & =\alpha([0, r] \cap A) \cup([r, \infty) \cap A)] \\
& =\alpha([0, r] \cap A) \cup \alpha([r, \infty) \cap A) .
\end{aligned}
$$

Since $[0, r] \cap A$ is compact, $\alpha([0, r] \cap A)$ is compact, thus closed, and by the first case $\alpha([r, \infty) \cap A)$ is closed. We now have $\alpha$ is a closed bijection and thus an isomorphism.

Let $n$ be an integer larger than 1 such that the lemma is true for all nonnegative integers less than $n$. Let $S$ denote $\left(R^{x}\right)^{n}$, and define $\hat{\alpha}: E(S) \rightarrow E(S)$ by $\hat{\alpha}(e)=\alpha(e)$ for all $e$ in $E(S)$. Since $\hat{\alpha}$ is bijective and since $E(S)$ is finite, $\hat{\alpha}$ is an isomorphism. For each $e$ in $E(S)$ define $\psi_{e}: S \rightarrow e S$ by $\psi_{e}(s)=e s$ for all $s$ in $S$. Let $e_{1}=(0,1,1, \cdots, 1)$ and $e_{2}=(1,0,0, \cdots, 0)$, and let $A=\psi_{e_{1}}^{-1}\left(e_{1}\right)$ and $B=\psi_{e_{2}}^{-1}\left(e_{2}\right)$. Then $A \cong R^{x}, B \cong\left(R^{x}\right)^{n-1}$ and $e_{1} \cdot e_{2}=0$. Define $F: A \times B \rightarrow S$ by $F(a, b)=a b$; then, by Lemma $4.4 \mathrm{i}, F$ is an isomorphism. Let $f_{1}=\alpha\left(e_{1}\right)$ and $f_{2}=$ $\alpha\left(e_{2}\right)$. We now show $\alpha(A)=\psi_{e_{1}}^{-1}\left(f_{1}\right) \cong R^{x}, \alpha(B)=\psi_{f_{2}}^{-1}\left(f_{2}\right) \cong\left(R^{x}\right)^{n-1}$, and $\alpha(A) \cap \alpha(B)=\{1\}$. From which it will follow by Lemma 4.4i that the morphism $G: \alpha(A) \times \alpha(B) \rightarrow S$, defined by $G(a, b)=a b$, is an isomorphism. Let $A_{1}=\psi_{f_{1}}^{-1}\left(f_{1}\right)$ and $A_{2}=\psi_{f_{2}}^{-1}\left(f_{2}\right)$. Since $\hat{\alpha}$ is an isomorphism, $\hat{\alpha}$ preserves the less than order on $E(S)$; thus $\operatorname{dim} H\left(f_{1}\right)=\operatorname{dim} H\left(e_{1}\right)=n-1$ 
and $\operatorname{dim} H\left(f_{2}\right)=\operatorname{dim} H\left(e_{2}\right)=1$. Therefore, $A_{1} \cong R^{x}$ and $A_{2} \cong\left(R^{x}\right)^{n-1}$ (Lemma 4.4iia). If $A_{1} \cap A_{2} \neq\{1\}$, then either $f_{1} \in A_{1} \cap A_{2}$ or there is an element $g \in H(1) \cap A_{1} \cap A_{2}$ with $g \neq 1$. Since $f_{1} \cdot f_{2}=\alpha\left(e_{1}\right) \cdot \alpha\left(e_{2}\right)=$ $\alpha\left(e_{1} e_{2}\right)=\alpha(0)=0, f_{1} \notin A_{2}$, and thus there is a $g \in H(1) \cap A_{1} \cap A_{2}$ with $g \neq 1$. Since $A_{1} \cong R^{x}$ either $\left\{g^{n}\right\}_{n=1}^{\infty}$ converges to $f_{1}$ or $\left\{\left(g^{-1}\right)^{n}\right\}_{n=1}^{\infty}$ converges to $f_{1}$ [3]. But both imply $f_{1} \in A_{2}$ which is impossible by the above. Thus $A_{1} \cap A_{2}=\{1\}$. Clearly, $\alpha(A) \subseteq A_{1}$. Let $t \in A_{1}$. Since $\alpha(S)=\alpha(A \cdot B)=\alpha(A) \cdot \alpha(B)$, there is an element $a \in \alpha(A)$ and $b \in \alpha(B)$ such that $t=a b$. It follows that $f_{1}=f_{1} t=f_{1} a \cdot b=f_{1} b$ which implies $b \in A_{1}$. But $\alpha(B) \subseteq B_{1}$ and $B_{1} \cap A_{1}=\{1\}$; thus $b=\{1\}$. The proof that $\alpha(B)=B_{1}$ is similar and will therefore be omitted. We now have the following commutative diagram:

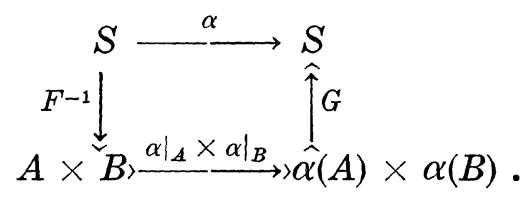

By the inductive hypothesis, $\left.\alpha\right|_{A}: A \rightarrow \alpha(A)$ and $\left.\alpha\right|_{B}: B \rightarrow \alpha(B)$ are isomorphisms. The lemma now follows.

Lemma 4.6. Let $X, Y$ and $Z$ be Hausdorff spaces and assume $F: X \times Y \rightarrow Z$ is a continuous surjection. If there are continuous surjections $\alpha: Z \rightarrow X$ and $\beta: Z \rightarrow Y$ such that the diagram

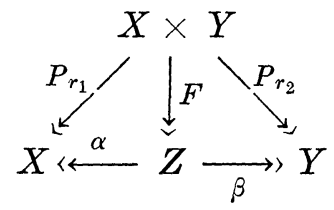

is commutative, then $F$ is a homeomorphism.

Proof. The inverse of $F$ is given by $z \mapsto(\alpha(z), \beta(z))$ which is clearly continuous.

THEOREM 4.7. If $S$ is an object in both $\mathscr{R}$ and $\mathscr{S}_{1}$, then $S \cong\left(R^{x}\right)^{n}$. where $n=\operatorname{dim} H_{S}(1)$.

Proof. The proof is by induction on $\operatorname{dim} H(1)$. The claim for $\operatorname{dim} H(1)=1$ is proven in [5]. Let $n$ be an integer larger than 1 such that the claim is true for all positive integers less than $n$. Let $e$ be an idempotent with $e>0$ and $e S \cong R^{x}$ (Corollary 3.2). By Lemma 4.3 there is an idempotent $f$ with $f \neq 0, \operatorname{dim} H(f)=n-1$ and $e f=0$. Let $A=\psi_{f}^{-1}(f)$ and $B=\psi_{e}^{-1}(e)$. Then by the inductive hypothesis, 
$A \cong R^{x}$ and $B \cong\left(R^{x}\right)^{n-1}$. Also $\psi_{e}^{-1}(H(e)) \cong H(e) \times B \cong R_{+} \times B$ (Proposition 3.4). Now define a morphism $F: A \times B \rightarrow S$ by $F(a, b)=a b$. Observe that $\psi_{e}(F(a, b))=e a b=e a$ and $\psi_{f}(F(a, b))=f b$. We now show $S=A \cdot B$. Since $E(S) \cong Z^{n}$ it follows that $E(S)=E(A) \cdot E(B)$. Let $s \in S$; then $\delta(s)=e_{1} \cdot f_{1}$ for some $e_{1} \in E(A)$ and $f_{1} \in E(B)$. Also, $s=\delta(s) \cdot g$ for some $g \in H_{S}(1)$. Since $H_{A}(1) \cap H_{B}(1)=\{1\}$ (see proof that $A_{1} \cap B_{1}=$ $\{1\}$ in Lemma 4.5), $g=a \cdot b$ for some $a \in H_{A}(1)$ and $b \in H_{B}(1)$. Thus $s=\delta(s) g=\delta(s) a b=e_{1} f_{1} a b=\left(e_{1} a\right)\left(f_{1} b\right) \in A \cdot B$. Clearly, $\psi_{e}(A)=e A \subseteq e S$. Let $t \in e S$; then $t=e a \cdot b$ for some $a \in A$ and $b \in B$. Thus $t=e a b=$ $e b \cdot a=e a$ and hence $e A=e S$. By Lemma 4.5, $\left.\psi_{e}\right|_{A}: A \rightarrow e S$ is an isomorphism. Similarly it can be shown that $f B=f S$ and thus, by Lemma 4.5, $\left.\psi_{f}\right|_{B}: B \rightarrow f S$ is an isomorphism. We now have the following diagram

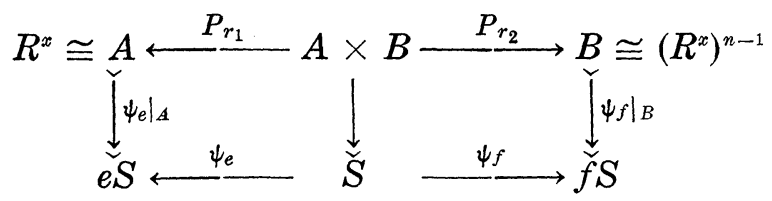

which can be reduced to

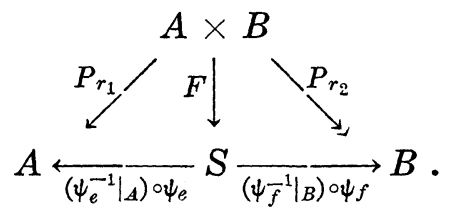

Thus by Lemma 4.6, $F$ is an isomorphism, and the theorem now follows by induction.

Definition. An object $S$ in $\mathscr{S}$ is an $H$-semigroup if (i) $H_{S}(1) \cong R_{+}$ and (ii) $K(S)$ is compact.

Lemma 4.8. Let $S$ be a object in $\mathscr{S}_{1}$ having the added properties that (i) $H_{S}(1)$ is a real vector group of dimension $n$ and (ii) $K(S)$ is compact. Then there are subsemigroups $S_{1}, \cdots, S_{n}$ of $S$ which are $H$-semigroups, the morphism $m: \mathbf{X}_{i=1}^{n} S_{i} \rightarrow S$ defined by $m\left(\left(s_{1}, \cdots, s_{n}\right)\right)=$ $s_{1} \cdot s_{2} \cdot \cdots \cdot s_{n}$ is a surmorphism which preserves the H-class structure of $\mathrm{X}_{i=1}^{n} S_{i}$, and also $m$ induces an isomorphism on the groups of units. Further, for each $i$ there is an idempotent $e_{i}$ with $\operatorname{dim} H\left(e_{i}\right)=n-1$ and $S_{i}=\psi_{e_{i}}^{-1}\left(H\left(e_{i}\right)_{c}\right)$.

Proof. Since $E(S) \cong Z^{n}$, there are exactly $n$-idempotents $e_{1}, \cdots, e_{n}$ in $S$ with $\operatorname{dim} H\left(e_{i}\right)=n-1$. By Proposition 3.4 and since $H_{S}(1)$ is a real vector group, each $\psi_{e_{i}}^{-1}\left(H\left(e_{i}\right)_{c}\right)$, is an $H$-semigroup. Let $S_{i}=$ $\psi_{e_{i}}^{-1}\left(H\left(e_{i}\right)_{c}\right)$, and let $F: S \rightarrow\left(R^{x}\right)^{n}$ be a surmorphism which preserves 
the $H$-class structure of $S$ (Proposition 3.11 then Theorem 4.7). Since $F$ preserves the $H$-class structure of $S, \operatorname{dim} H\left(e_{i}\right)=\operatorname{dim} H\left(F\left(e_{i}\right)\right)=n-1$ for $i=1,2, \cdots, n$ and, also, $F\left(S_{i}\right)=\psi_{e_{i}^{1}}^{-1}\left(H\left(e_{i}^{1}\right)\right) \cong R^{x}$ for $i=1,2, \cdots, n$, where $e_{i}^{1}=F\left(e_{i}\right)$. Using the structure of $\left(R^{x}\right)^{n}$ we know $\psi_{e_{i}}^{-1}\left(F\left(e_{i}^{1}\right)\right) \cong R^{x}$ if and only if there is an integer $j(i), 1 \leqq j(i) \leqq n$ such that

$$
P_{r_{j(i)}} \mid \psi_{\epsilon_{i}^{1}}^{-1}\left(F\left(e_{i}^{1}\right)\right): \psi_{\epsilon_{i}^{1}}^{-1}\left(F\left(e_{i}^{1}\right)\right) \rightarrow R^{x}
$$

is an isomorphism. For each $i, i=1,2, \cdots, n$ let $\pi_{i}: S_{i} \rightarrow S_{i} / K\left(S_{i}\right)$ be the natural map where $S / K\left(S_{i}\right)$ denotes the Rees quotient semigroup. Since each $K\left(S_{i}\right)$ is compact [3], $\pi_{i}$ is a closed map. Thus for each $i$ there is a bijective morphism $\beta_{i}: S_{i} \rightarrow R^{x}$ such that the following diagram commutes

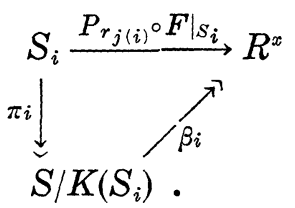

By Lemma 4.5 each $\beta_{i}$ is an isomorphism. Since each $K\left(S_{i}\right)$ is compact, it is easy to show that a net $\left\{g_{\alpha}\right\}_{\alpha \in A} \subseteq S_{i}$ has a cluster point if and only if $\left\{\pi_{i}\left(g_{\alpha}\right)\right\}_{\alpha \in A}$ has a cluster point. Thus it follows that $\left\{g_{\alpha}\right\}_{\alpha \in A} \subseteq S_{i}$ has a cluster point if and only if $\left\{P_{r_{j(i)}}\left(F\left(g_{\alpha}\right)\right)\right\}_{\alpha \in A}$ has a cluster point.

Let $x \in S$ and let $\left\{g_{\alpha}\right\}_{\alpha \in A}$ be a net in $H_{S}(1)$ which converges to $x$. Then for each $\alpha$ there are elements $g_{i}(\alpha) \in S_{i} i=1,2, \cdots, n$ such that $g_{\alpha}=g_{1}(\alpha) \cdot g_{2}(\alpha) \cdot \cdots \cdot g_{n}(\alpha)$. Since $P_{r_{j(i)}} F\left(g_{i}(\alpha)\right)=P_{r_{j(i)}}\left(F\left(g_{\alpha}\right)\right)$ for $i=$ $1,2,3, \cdots, n$ and since $P_{r_{j(i)}}\left(F\left(g_{\alpha}\right)\right)$ has a cluster point and by the above, each $\left\{g_{i}(\alpha)\right\}_{\alpha \in A}$ has a cluster point. Clearly, we can choose a subnet $\left\{g_{\alpha}\right\}_{\alpha \in B}$ such that each $\left\{g_{i}(\alpha)\right\}_{\alpha \in B}$ converges. It now follows that $x \in m\left(\mathbf{X}_{i=1}^{n} S_{i}\right)$. Clearly, $m$ induces an isomorphism on the groups of units.

THEOREM 4.9. Let $S \in \mathscr{S}_{1}$. Then $S \cong T \times \boldsymbol{R}^{n}$ for a suitable $n$ and where $T$ is an object in $\mathscr{S}_{1}$ satisfying the following: There are subsemigroups $S_{1}, \cdots, S_{n}$ of $T$ with each $S_{i}$ an $H$-semigroup and a surmorphism $m: H_{T}(1)_{c} \times\left(\mathbf{X}_{i=1}^{n} S_{i}\right) \rightarrow T$ which preserves the H-class structure and which induces an isomorphism on the groups of units. Further, there are surmorphisms $G_{1}: S \rightarrow\left(R^{x}\right)^{n}$ and $G_{2}: H_{T}(1)_{c} \times\left(\mathbf{X}_{i=1}^{n} S_{i}\right) \rightarrow\left(R^{x}\right)^{n}$ such that the following diagram is commutative

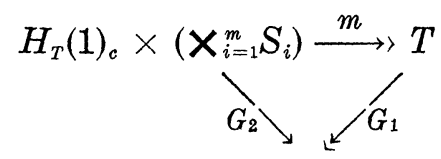

$\left(R^{x}\right)^{n}$ 
Proof. By Proposition 3.5, $S \cong T \times \boldsymbol{R}^{m}$ for a suitable choice of $m$, where $T \in \mathscr{S}$ with $K(T) \in \mathscr{C}_{c}$. Since $E(S) \cong Z^{n}$ for some $n$ and since $E(S) \cong E(T), T \in \mathscr{S}_{1}$. Using Lemma 3.1 and Corollary 3.2, it is easy to see that $\operatorname{dim} H_{T}(1)=n$. Since $E(S) \cong Z^{n}$, there are exactly $n$ idempotents $e_{1}, \cdots, e_{n}$ such that $\operatorname{dim} H\left(E_{i}\right)=n-1$. For each $e_{i}$ let $C_{i}$ be a compact subgroup of $H\left(e_{i}\right)_{c}$ which is open relative to $H\left(e_{i}\right)_{c}$. It follows from Proposition 3.4 and the fact that each $H\left(e_{i}\right)$ is open in $T \backslash H_{T}(1)$, that each $\psi_{e_{i}}^{-1}\left(C_{i}\right)$ is a locally compact semigroup which contains a dense group whose complement is compact. Since each $\psi_{e_{i}}^{-1}\left(C_{i}\right) \in \mathscr{S}$ and by [7], there is a one-parameter subgroup $P_{i} \cong \psi_{e_{i}}^{-1}\left(C_{i}\right) \cap H_{T}(1)$ such that $\bar{P}_{i} \cap C_{i} \neq \varnothing$. For each $i$ let $S_{i}=\bar{P}_{i}$; then each $S_{i}$ an $H$-semigroup. Let $m: H_{T}(1)_{c} \times\left(\mathrm{X}_{i=1}^{n} S_{i}\right) \rightarrow T$ be a morphism defined by $m\left(g, s_{1}, \cdots, s_{n}\right)=$ $g \cdot s_{1} \cdot s_{2} \cdot \cdots \cdot s_{n}$ and let $m_{1}: \mathrm{X}_{i=1}^{n} S_{i} \rightarrow T$ be the morphism defined by $m_{1}(s)=m(1, s)$ for all $s$ in $X_{i=1}^{n} S_{i}$.

Let $T / R$ be the semigroup constructed as in Theorem 3.9 and let $F: T \rightarrow T / R$ be the natural map. Since $F$ preserves the $H$-class structure, $\operatorname{dim} H\left(F\left(e_{i}\right)\right)=n-1$ for each $i$. Since for each $i F\left(K\left(S_{i}\right)\right)$ is a compact ideal for $\left.F\left(P_{i}\right), \overline{F\left(P_{i}\right)}=F\left(P_{i}\right) \cup F\left(K / S_{i}\right)\right)$ [5]; thus $F\left(S_{i}\right)=\overline{F\left(P_{i}\right)}$. Also, $H\left(F\left(e_{i}\right)\right)_{c}$ is a compact ideal for $F\left(P_{i}\right)$; thus $F\left(S_{i}\right)=\overline{F\left(P_{i}\right)}=$ $F\left(P_{i}\right) \cup H\left(F\left(e_{i}\right)\right)_{c}$. It now follows from Lemma 4.8 that $F\left(m_{1}\left(\mathbf{X}_{i=1}^{n} S_{i}\right)\right)=$ $T / R$ and thus $m_{i}\left(\mathbf{X}_{i=1}^{n} S_{i}\right) \cdot H_{T}(1)=T$. Therefore, $m$ is a surmorphism.

Let $T_{1}=\overline{m_{1}\left(\mathbf{X}_{i=1}^{n} S_{i}\right)}$. Since $E(T)=E\left(m_{i}\left(\mathbf{X}_{i=1}^{n} S_{i}\right)\right) \cong Z^{n}, E(T) \cong Z^{n}$, and thus it follows that $\operatorname{dim} H_{T_{1}}(1)=n$. Let $F_{1}: T_{1} \rightarrow T_{1} / R_{1}$ be the natural map where $T_{1} / R_{1}$ is the semigroup guaranteed by Theorem 3.9. Let $H_{1}=H_{T_{1}} / R_{1}(1)$. Then $H_{1}$ is an $n$-dimensional vector group with $\left.\overline{F_{1}\left(m_{1}\left(\mathbf{X}_{i=1}^{n} P_{i}\right)\right.}\right)=H_{1}$. Thus by $P_{2}$ there is a morphism $\beta: H_{1} \rightarrow \mathbf{X}_{i=1}^{n} P_{i}$ such that $F_{1} m_{1} \beta=I_{T_{1} / R_{1}}$. It follows that the inverse of $\left.F_{1}\right|_{m_{1}}\left(\mathbf{X}_{i=1}^{n} P_{i}\right)$ is the corestriction of $m_{1} \beta$ to $m_{1}\left(\mathbf{X}_{i=1}^{n} P_{i}\right)$. Thus $m_{1}\left(\mathbf{X}_{i=1}^{n} P_{i}\right)$ is a locally compact subgroup $H_{T_{1}}(1)$ and thus closed. Therefore, it follows that the corestriction of $m_{1} \mid \mathbf{X}_{i=1}^{n} P_{i}: \mathbf{X}_{i=1}^{n} P_{i} \rightarrow \mathbf{X}_{i=1}^{n} P_{i}$ is an isomorphism. Since $H_{T}(1)=m_{i}\left(\mathbf{X}_{i=1}^{n} P_{i}\right) \cap H_{T}(1)_{c}$ and $m_{1}\left(\mathbf{X}_{i=1}^{n} P_{i}\right) \cap H_{T}(1)_{c}=\{1\}$, it now easily follows that $m$ induces an isomorphism on the group of units.

The remainder of the proof follows directly from Theorem 3.11 and Theorem 4.7.

The author wishes to thank the referee for his many helpful suggestions. In particular, the author wishes to thank the referee for his suggestions on the order in which the results should be presented.

\section{REFERENCES}

1. A. H. Clifford and G. B. Preston, The algebraic theory of semigroups, Amer. Math. Soc., Providence, 1961.

2. E. Hewitt and K. A. Ross, Abstract hormonic analysis, Academic Press, New York, 1963. 
3. K. H. Hofmann, Locally compact semigroups in which a subgroup with compact complement is dense, Trans. Amer. Math Soc. 106 (1965), 19-51.

4. K. H. Hofmann and P. S. Mostert, Elements of compact semigroups, Charles E. Merrill Books, Columbus, 1966.

5. J. G. Horne, Jr., The boundary of a one-parameter group in a semigroup, Duke Math. J. 31 (1964), 109-117.

6. - Semigroups of a half-space, (to appear)

7. J. W. Stepp, D-semigroups, Proc. Amer. Math. Soc. 22 (1969), 402-406.

8. T. S. Wu, Locally compact semigroups with dense maximal subgroups, Trans. Amer. Math. Soc. 113 (1964), 151-168.

Received March 12, 1969, and revised form October 31, 1969. This research was partially supported by NSF Grant GP 8780.

UNIVERSITY OF HOUSTON

Houston, TeXas 


\section{PACIFIC JOURNAL OF MATHEMATICS}

EDITORS

H. SAMELSON

Stanford University

Stanford, California 94305

RichaRd PIERCE

University of Washington

Seattle, Washington 98105
J. DUGUNDJI

Department of Mathematics

University of Southern California

Los Angeles, California 90007

RICHARD ARENS

University of California

Los Angeles, California 90024

\section{ASSOCIATE EDITORS}

E. F. BECKENBACH

B. H. NeumanN

F. WOLF

K. YOSHIDA

\section{SUPPORTING INSTITUTIONS}

UNIVERSITY OF BRITISH COLUMBIA

CALIFORNIA INSTITUTE OF TECHNOLOGY

UNIVERSITY OF CALIFORNIA

MONTANA STATE UNIVERSITY

UNIVERSITY OF NEVADA

NEW MEXICO STATE UNIVERSITY

OREGON STATE UNIVERSITY

UNIVERSITY OF OREGON

OSAKA UNIVERSITY

UNIVERSITY OF SOUTHERN CALIFORNIA
STANFORD UNIVERSITY

UNIVERSITY OF TOKYO

UNIVERSITY OF UTAH

WASHINGTON STATE UNIVERSITY

UNIVERSITY OF WASHINGTON

AMERICAN MATHEMATICAL SOCIETY CHEVRON RESEARCH CORPORATION TRW SYSTEMS

NAVAL WEAPONS CENTER 


\section{Pacific Journal of Mathematics}

May, 1970

Johan Aarnes, Edward George Effros and Ole A. Nielsen, Locally compact

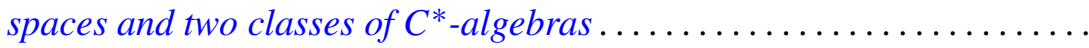

Allan C. Cochran, R. Keown and C. R. Williams, On a class of topological

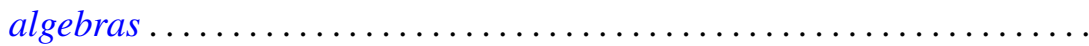

John Dauns, Integral domains that are not embeddable in division rings ....

Robert Jay Daverman, On the number of nonpiercing points in certain

crumpled cubes.....................................

Bryce L. Elkins, Characterization of separable ideals ................

Zbigniew Fiedorowicz, A comparison of two naturally arising uniformities

on a class of pseudo-PM spaces ...........................

Henry Charles Finlayson, Approximation of Wiener integrals of functionals

continuous in the uniform topology ........................

Theodore William Gamelin, Localization of the corona problem ...........

Alfred Gray and Paul Stephen Green, Sphere transitive structures and the

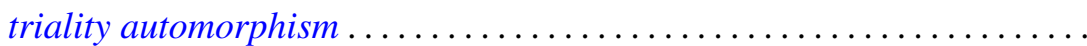

Charles Lemuel Hagopian, On generalized forms of aposyndesis ..........

J. Jakubík, On subgroups of a pseudo lattice ordered group ...............

Cornelius W. Onneweer, On uniform convergence for Walsh-Fourier

series..................................

Stanley Joel Osher, On certain Toeplitz operators in two variables ...

Washek (Vaclav) Frantisek Pfeffer and John Benson Wilbur, On the

measurability of Perron integrable functions............

Frank J. Polansky, On the conformal mapping of variable regions...

Kouei Sekigawa and Shûkichi Tanno, Sufficient conditions for a Riemannian manifold to be locally symmetric ...................

James Wilson Stepp, Locally compact Clifford semigroups ....

Ernest Lester Stitzinger, Frattini subalgebras of a class of solvable Lie

algebras ................................

George Szeto, The group character and split group algebras...

Mark Lawrence Teply, Homological dimension and splitting torsion

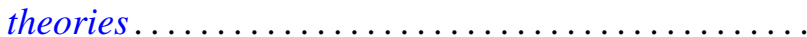

David Bertram Wales, Finite linear groups of degree seven. II ...

Robert Breckenridge Warfield, Jr., An isomorphic refinement theorem for

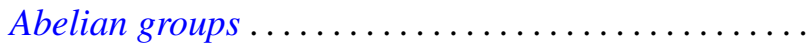

James Edward West, The ambient homeomorphy of an incomplete subspace

of infinite-dimensional Hilbert spaces................

Peter Wilker, Adjoint product and hom functors in general topology ...

Daniel Eliot Wulbert, A note on the characterization of conditional 